\title{
Creencias Epistemológicas de Estudiantes de Secundaria en la Aceptación y Uso de Facebook
}

\author{
Epistemological Beliefs on Secondary Students in the \\ Acceptance and Use of Facebook
}

\author{
Mario Armando Cartagena-Beteta ${ }^{1 *}$ \\ Lucrecia Chumpitaz-Campos ${ }^{2}$ \\ ${ }^{1}$ Pontificia Universidad Católica del Perú, Perú \\ ${ }^{2}$ Universidad Femenina del Sagrado Corazón, Perú
}

\begin{abstract}
Las creencias de los alumnos para efectos de aprendizaje, evolucionan con su edad y experiencias; lo que permite explicar la aceptación y uso de un medio didácticotecnológico. De esta manera, se buscó: comprender la relación entre las creencias epistemológicas y la aceptación y uso de Facebook y describir las categorías finales que constituyen las creencias epistemológicas de los estudiantes en sus experiencias de aprendizaje con la red social en cuestión. Se aplicó un enfoque fenomenográfico a partir de entrevistas semiestructuradas y en profundidad. La muestra estuvo constituida por 10 estudiantes de secundaria, quienes desarrollaron actividades de aprendizaje en Facebook a lo largo del año lectivo 2018. Los resultados indican que las creencias de los estudiantes respecto de las posibilidades de aprendizaje en el entorno de la página de Facebook "Entorno Virtual de Religión” constituyen predictores válidos para su aceptación y uso en el área de educación religiosa. En efecto, se identificó que la facilidad, el tiempo invertido, la influencia social, así como los beneficios académicos y de aprendizaje constituyen las creencias de los alumnos para aceptar y usar la página en cuestión. Se sugiere realizar estudios similares bajo un paradigma mixto con diseños transversales y/o longitudinales, en orden a fortalecer el marco teórico de la integración curricular de las TIC.
\end{abstract}

Descriptores: Creencia; Epistemología; Redes sociales; Informática educativa; Educación religiosa; Investigación pedagógica.

Students' beliefs for learning purposes, evolve with their age and experiences; what allows to explain the acceptance and use of a didactic-technological means. In this way, we sought to: Understand the relationship between epistemological beliefs and the acceptance and use of Facebook and describe the final categories that constitute students' epistemological beliefs in their learning experiences with the social network in question. A phenomenological approach was applied based on semistructured and in-depth interviews. The sample consisted of 10 secondary school students, who had the experience of developing learning activities on Facebook throughout the 2018 school year. The results indicate that the beliefs of the students regarding the possibilities of learning in the environment of The Facebook page "Virtual Environment of Religion" are valid predictors for acceptance and use in the area of religious education. Indeed, it was identified that the facility, the time invested, the social influence, as well as the academic and learning benefits constitute the students' beliefs to accept and use the page in question. It is suggested to carry out similar studies under a mixed paradigm with transversal and / or longitudinal designs, in order to strengthen the theoretical framework of ICT curricular integration.

Keywords: Belief; Epistemology; Social networks; Computer uses in education; Religious education; Educational research.

*Contacto: mcartagenab@pucp.pe

ISSN: 1696-4713

www.rinace.net/reice/

revistas.uam.es/reice
Recibido: 19 de julio 2019

$1^{\text {a }}$ Evaluación: 29 de septiembre 2019

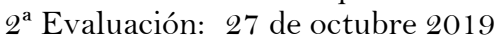

Aceptado: $\quad 7$ de diciembre 2019 


\section{Introducción}

Desde el trabajo de Clark y Peterson (1984) titulado procesos de pensamiento en el profesor, se apertura un campo de estudio sobre las creencias de los docentes. Así, irrumpe en la investigación, el paradigma del pensamiento docente, según el cual "el profesor y el alumno son agentes activos, con creencias y percepciones personales que, de algún modo, influyen y determinan su conducta" (Prieto, 2007, p. 37). Este paradigma, encuentra en Argyris y Schon (1974) un referente teórico, según el cual, las personas son agentes que actúan intencionadamente sobre su realidad y planifican sus acciones posteriores a partir de las experiencias que van obteniendo. De allí que, existen trabajos que indican que las concepciones y creencias sobre la enseñanza y el aprendizaje en diversos contextos se interrelacionan (Aypay, 2010; Kizilgunes, Tekkaya y Sungur, 2009; Lodewyk, 2007; Yilmaz-Tuzun y Sami, 2010). Por ejemplo, los estudios señalan que las creencias de los estudiantes sobre el éxito o fracaso afectan su esfuerzo y rendimiento; así también, sus creencias sobre la lectura y materiales de texto influyen en la conducta y procesamiento de la información (Buehl y Alexander, 2001).

En este estudio nos centraremos en los estudiantes. Comprenderemos cómo sus creencias, específicamente las epistemológicas, pueden constituirse en predictores para aceptar y usar una página de Facebook en el área curricular de educación religiosa. En este sentido, se busca incluir la voz de los estudiantes en las decisiones que toman los educadores para innovar y mejorar su práctica pedagógica con el uso de la tecnología (Stefl-Mabry, Radlick y Doane, 2010). Es sabido que algunas investigaciones ignoran las motivaciones personales y puntos de vista de los alumnos cuando deciden realizar o no un comportamiento en particular (Al-Azawei, 2018).

Cabe señalar que el área de educación religiosa se trabaja en las escuelas públicas del Perú de acuerdo con el Decreto Ley 23211. Por tanto, la enseñanza de esta asignatura propone desarrollar competencias en (1) Comprensión Doctrinal cristiana, entendida como la capacidad de "conocer, comprender y ser capaz de aplicar las enseñanzas que se recogen de las fuentes doctrinales para que pueda formar su conciencia moral" (Ministerio de Educación, 2015, p. 437) y (2) Discernimiento de Fe, que "busca que los estudiantes desarrollen su capacidad reflexiva y analítica frente a los acontecimientos de la vida y las situaciones para actuar de manera coherente con la fe y ser testimonio de vida cristiana" (Ministerio de Educación, 2015, p. 437). De esta manera, el aprendizaje se entiende como "el desarrollo de capacidades en el estudiante que lo hacen competente para seguir descubriendo y aprendiendo, para saber actuar frente a nuevas posibilidades, buscando desarrollar su pensamiento crítico y reflexivo, el discernimiento de los hechos de la vida cotidiana y la resolución de problemas" (Ministerio de Educación, 2010, p. 49).

\section{Revisión de la literatura}

\subsection{Creencias epistemológicas}

De acuerdo con Pajares (1992), así como a Buehl y Alexander (2001), somos conscientes de la complejidad que supone estudiar las creencias. Sin embargo, es necesario delimitar su significado, examinar los principios que las sustentan y evaluarlas de tal manera que puedan constituirse en un campo significativo para la investigación pedagógica (Cvetkova et al., 2015). De hecho, los estudios sobre creencias en estudiantes están manifestando que existe relación con factores como contenido didáctico, percepciones del ambiente de 
aprendizaje, el conjunto de tareas académicas y sus características personales (YilmazTuzun y Sami, 2010). Por tanto, conceptualizar las creencias implica tener claro a qué tipo de creencias nos referimos, así como las necesidades y exigencias de la investigación (Prieto, 2007). De allí que entendemos por creencias a las "disposiciones a interpretar la propia experiencia, las cuales constituyen una base para guiar la acción futura de un modo determinado" (Samuelowicz, 1999, p. 9). Específicamente, nos interesan las creencias epistemológicas, las cuales se entienden dependiendo del objetivo del investigador. Así, cuando se les manipula para mejorar un problema de aprendizaje se comprenden en términos simples y unidimensionales focalizados en un área en cuestión (por ejemplo, matemática, ciencias, entre otros). Sin embargo, cuando la investigación se centra en la forma cómo evolucionan junto con el desarrollo intelectual de acuerdo a las diferentes etapas del aprendizaje, entonces se plantean modelos de creencias epistemológicas (Schommer, 1994).

La evolución de estos modelos nos remite a Perry (1968, 1970), Kitchener y King (1981) y Belenky y colaboradores (1986). Perry (1968) trabajó un modelo de desarrollo epistemológico adulto administrando cuestionarios y realizando entrevistas con alumnos de la universidad de Harvard. Con esos datos, concluyó que los estudiantes ingresan a la universidad creyendo que el conocimiento es simple, seguro y transmitido por la autoridad; pero con el avance académico, sus convicciones entran en conflicto cambiando progresivamente. En esta línea, Kitchener y King (1981) continuaron con el trabajo de Perry y plantearon el "modelo del juicio reflexivo", según el cual existe una evolución en el conocimiento desde afirmaciones aceptadas por la fuerza de la autoridad de la que emanan (padres o maestros) hasta llegar a cuestionamientos que hacen redefinir las verdades aceptadas inicialmente. Lo trascendente es que los individuos con creencias epistemológicas básicas pueden dejar de comprender líneas sofisticadas de argumentación. Finalmente, Belenky y colaboradores (1986) se centraron en los puntos de vista de las mujeres sobre el conocimiento, la realidad y la autoridad. De allí que sugieren tener en cuenta creencias distintas a las de certeza y fuente de conocimiento.

La evolución de los modelos de creencias epistemológicas se detalla en la figura $1 \mathrm{y}$ permite comprender que la epistemología personal no es unidimensional. Esto significa que no se ha logrado captar la complejidad de la epistemología personal y se pueden enmascarar múltiples vínculos entre la epistemología personal y los diferentes aspectos del aprendizaje. En efecto, las creencias epistemológicas deberían ser tratadas como un sistema de creencias más o menos independientes. Por sistema se entiende, que no sólo hay por considerar una creencia a la vez y por independientes se indica que unas creencias pueden ser sofisticadas y otras no. Todas ellas afectan el aprendizaje. Por tanto, las creencias de los individuos sobre la fuente, certeza y organización del conocimiento, así como la velocidad y el control de la adquisición del conocimiento son preocupaciones clave de la epistemología personal e incluyen componentes afectivos y cognitivos (Schommer, 1994).

En síntesis, las creencias epistemológicas se definen como teorías de los alumnos sobre el conocimiento, su naturaleza y adquisición (Hofer y Pintrich, 1997). Por tanto, son consideradas como una construcción general de suposiciones sobre el conocimiento y su naturaleza (Harteis, Gruber y Hertramph, 2010) y estas son importantes para el aprendizaje puesto que coadyuvan a que los alumnos: (a) participen activamente en el aprendizaje; (b) persistan en las tareas; (c) comprendan el material escrito; y (d) afronten los problemas mal estructurados (Harteis, Gruber y Hertramph, 2010; Kizilgunes, 
Tekkaya y Sungur 2009; Lodewyk, 2007; Schommer, 1994). Sin embargo, existen dudas sobre: su relación con los campos del conocimiento (Buehl y Alexander, 2001); si son construcciones básicas generales; si pueden separarse de las suposiciones sobre los procesos de aprendizajes propios y ajenos; o si están ligadas al contexto y por tanto son situadas.

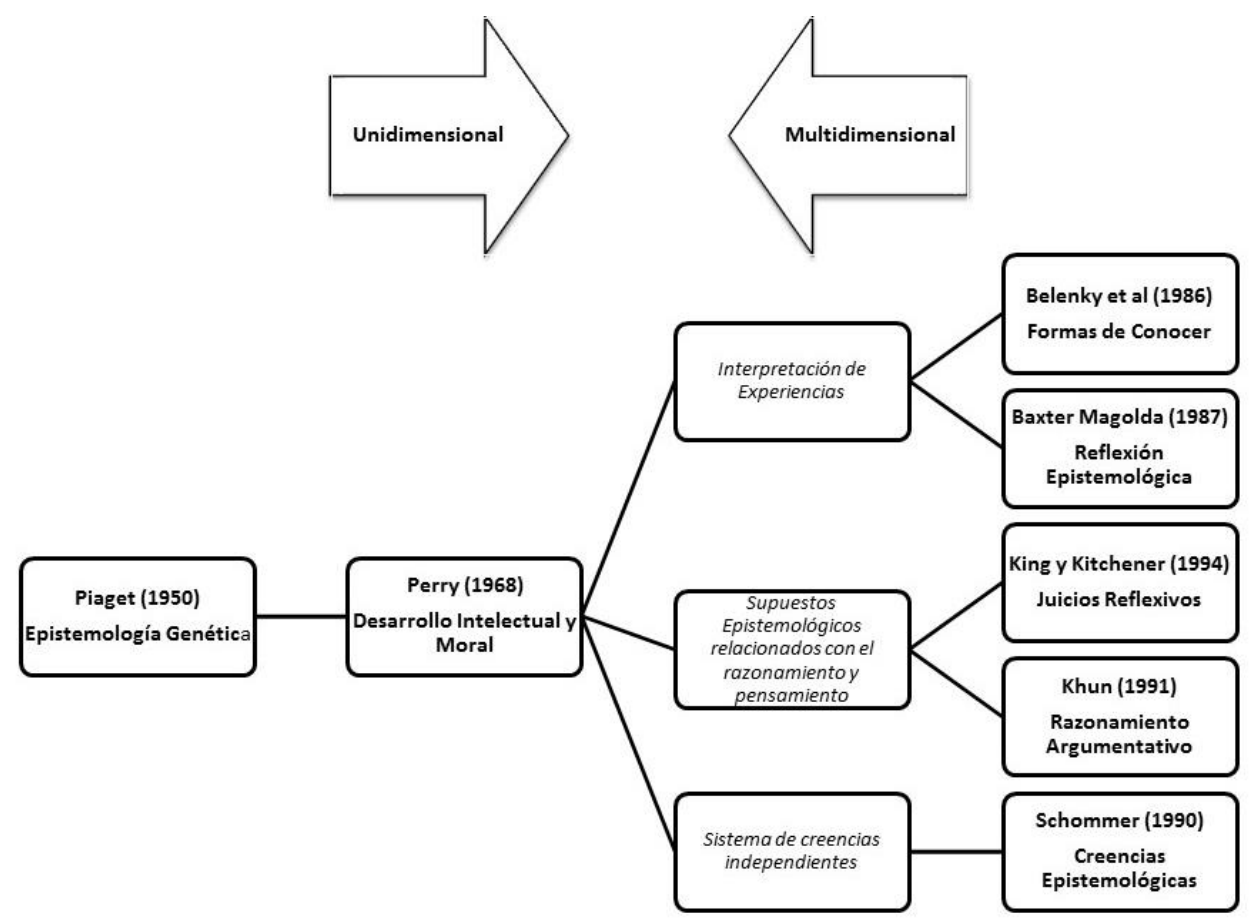

Figura 1. Evolución de las teorías epistemológicas

Fuente: Elaboración propia basada en Buehl y Alexander (2001), Hofer y Pintrich (1997) y

Lodewyk (2007).

A pesar de las dudas, cabe señalar que la construcción de un sistema de creencias epistémicas tiene implicaciones sustanciales en el aprendizaje (Harteis, Gruber y Hertramph, 2010; Schommer, 1998), por ejemplo: (1) los estudiantes, que creen que el aprendizaje ocurre rápidamente, tienden a leer textos más superficialmente; (2) los estudiantes, que creen que el conocimiento es cierto, tienden a aprender hechos de memoria en lugar de entender el significado de lo que debe ser aprendido; (3) los estudiantes, que creen que las capacidades de aprendizaje están determinadas por habilidades innatas, muestran menos interés en las actividades diseñadas para dominar desafíos complejos; y (4) los estudiantes que confían en las autoridades no tienden a desafiar las fuentes de información. Esto significa que las creencias de los estudiantes están asociadas con las conductas que demuestran en su proceso de aprendizaje.

\subsection{Teoría de la aceptación y uso de la tecnología}

La investigación sobre la aceptación de la tecnología ha generado un cuerpo completo de modelos que intentan explicar las razones de la adopción de tecnologías en diferentes campos de la actividad humana (Kim, 2015). De allí que se puede afirmar que es un área madura de estudio y reflexión (Venkatesh et al., 2003). Algunos de los modelos más significativos son la teoría de la acción razonada (Fishbein y Ajzen, 1975), el modelo de la aceptación tecnológica (Davis, 1989) y la teoría unificada de la aceptación y uso de las 
tecnologías (Venkatesh et al., 2003) cuyos postulados han sido usados en diferentes contextos y con distintos métodos de investigación de acuerdo a las exigencias de los estudios desarrollados (Williams, Rana y Dwivedi, 2014). En efecto, la necesidad de elegir entre diferentes modelos y teorías generó confusión entre los investigadores, por lo que Venkatesh y colaboradores (2003) desarrollaron un modelo unificado que reúne puntos de vista alternativos sobre el uso y la aceptación de una innovación (Taherdoost, 2018).

El trabajo de Venkatesh y colaboradores (2003) surgió con el objetivo de revisar y analizar la literatura de adopción de nuevas tecnologías de la información a partir de los principales modelos existentes, tal como se detalla en la figura 2. Para ello, compararon las similitudes y diferencias entre varios modelos existentes con lo que pudo validar empíricamente sus principios y formuló un modelo unificado. En efecto, integraron los elementos de ocho modelos teóricos: la teoría de la acción razonada (TRA); el modelo de aceptación tecnológica (TAM); el modelo motivacional (MM); la teoría de la conducta planificada (TPB); un modelo consensuado entre el modelo de la aceptación de la tecnología y la teoría del comportamiento planificado (C-TAM-TPB); el modelo de uso de PC (MPCU); la teoría de la difusión de la innovación (IDT) y la teoría social cognitiva (SCT).

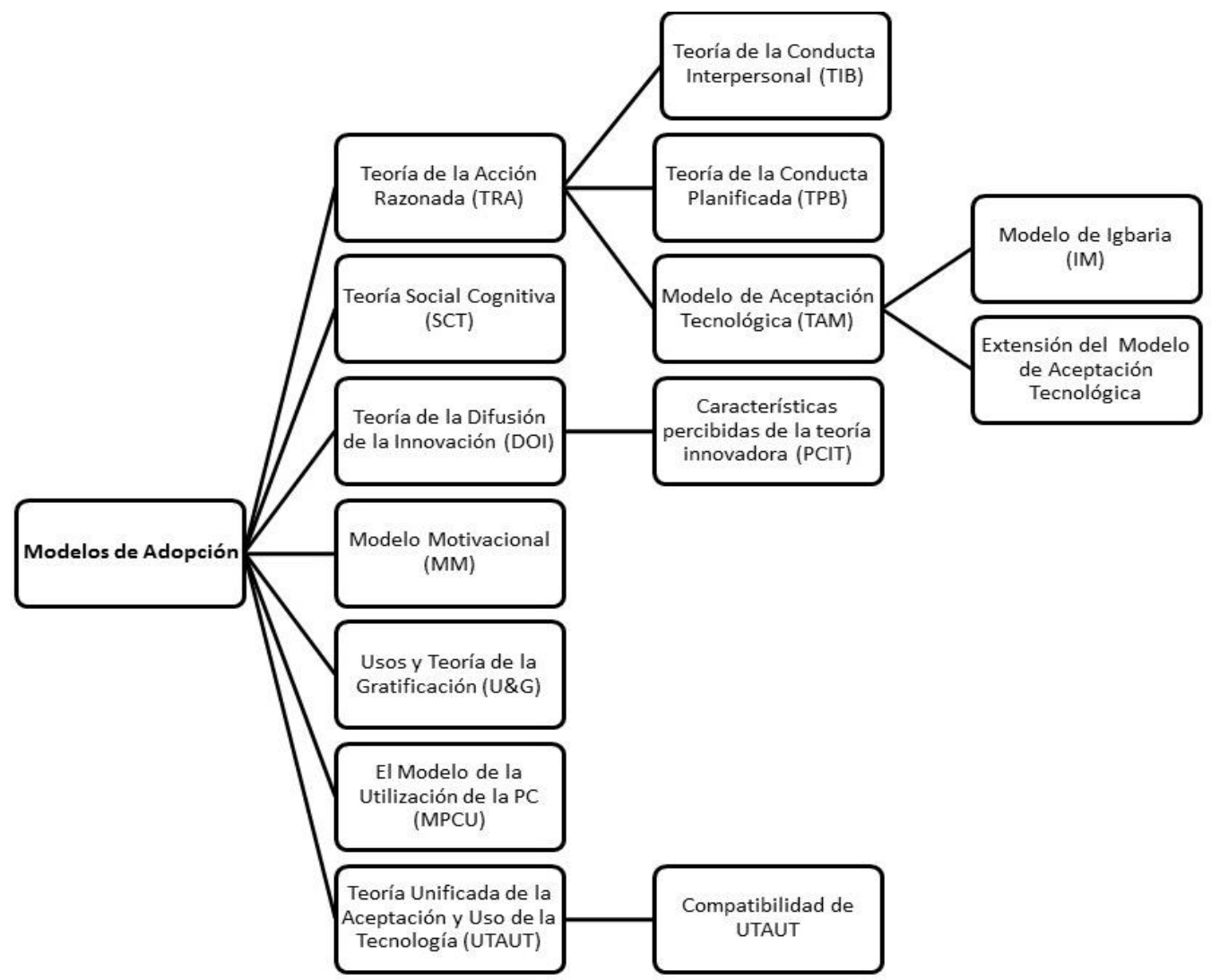

Figura 2. Evolución de los modelos teóricos sobre la adopción de tecnologías Fuente: Recuperado de Taherdoost (2018, p. 962).

La teoría unificada de la aceptación y uso de la tecnología (UTAUT) sugiere que cuatro constructos centrales (expectativa de rendimiento, expectativa de esfuerzo, influencia social y facilidad de condiciones) son directamente determinantes de la intención de comportamiento y que estos constructos son a su vez moderados por género, edad, experiencia y voluntad de uso (Venkatesh et al., 2003). Este modelo integrador ha 
permitido explicar la intención de conducta en el uso de la tecnología y el uso en sí. Sin embargo, la investigación de Williams y colaboradores (2011) indica que sólo el 3.6\% de un total de 450 artículos ha usado la teoría en su totalidad, mientras que la mayoría de investigadores ha integrado otros factores y usado las variables moderadoras de acuerdo a la conveniencia de sus estudios en curso (Lee, Korzan y Larsen, 2003). En efecto, Venkatesh, Thong y Xu $(2012,2016)$ plantearon una ampliación de su propuesta original integrando nuevos constructos como la motivación hedónica, el valor de precio y el hábito (UTAUT2), los cuales se centran en nuevas teorías ya que existían limitaciones prácticas para explicar la aceptación y uso de diferentes tecnologías en entornos organizacionales como no organizativos.

Venkatesh, Thong y Xu (2016) revisaron 1.267 estudios entre 2003 y 2014 clasificándolos en cuatro grupos: (1) artículos que usaron la teoría original; (2) artículos que aplicaron la teoría; (3) artículos que integraron la teoría con otras teorías; y (4) artículos que extendieron la teoría original. Con lo que se evidencia el robustecimiento del marco teórico a lo largo de los años. En esta línea, William, Rana y Dwivedi (2014) analizaron 174 artículos entre 2004 y 2011 encontrando que el modelo de aceptación tecnológica (TAM) ha sido el más usado en relación al modelo integrado UTAUT. Además, señalan que este modelo teórico ha sido usado sobre todo en gobierno electrónico (Zuiderwijk, Janssen y Dwivedi, 2015), banca electrónica, comercio electrónico (De Sena, Moriguchi, Andrade, 2016); debido a que se origina en el campo de la sociología, psicología y comunicaciones. En el campo educativo, se han registrado trabajos que en su conjunto buscan estudiar la reacción de los estudiantes frente a la tecnología y determinar los factores que contribuyen a su adopción y uso con fines de aprendizaje (Halili y Sulaiman, 2018; Nassuora, 2013; Raman, Sani y Kaur, 2014). Por tanto, esta investigación: (1) busca validar el uso de un entorno de aprendizaje diseñado en Facebook para el área de educación religiosa y (2) robustecer el marco teórico y experimental del uso del modelo UAUT en una realidad sudamericana.

\section{Método}

Los objetivos de esta investigación son: (1) Comprender la relación entre las creencias epistemológicas de los estudiantes de cuarto de secundaria de una I.E. pública de Lima y la aceptación y uso de Facebook en educación religiosa y (2) Describir las categorías finales que constituyen las creencias epistemológicas de los estudiantes en sus experiencias de aprendizaje con la página de Facebook "Entorno Virtual de Religión".

Se ha escogido un estudio cualitativo bajo el paradigma de investigación interpretativa. De acuerdo con Erickson (2012), la investigación cualitativa busca documentar en detalle la conducta de los eventos cotidianos e identificar los significados que tienen esos eventos para quienes participan en ellos y para quienes los presencian. Por tanto, este tipo de investigación es apropiada en educación si queremos conocer los matices de la comprensión subjetiva que motivan a los participantes en un entorno para ubicar y comprender el cambio a lo largo del tiempo.

Así mismo, siguiendo las sugerencias de los investigadores para este tipo de categorías de estudio (Cano y Cardelle-Elawar, 2004) se ha trabajado desde un diseño fenomenográfico, el cual tiene por objetivo identificar y describir las formas cualitativamente diferentes sobre cómo las personas experimentan (comprenden, perciben) fenómenos de su entorno 
(González-Ugalde, 2014, p. 144). Por tanto, el eje de la investigación no es el fenómeno o las personas, sino la relación entre ambos. Es decir, cómo el sujeto experimenta, percibe o comprende dicho fenómeno; de allí que el investigador debe poner entre paréntesis sus experiencias. Por tanto, es crucial que el sujeto del estudio haya experimentado el fenómeno de interés (Linder y Marshall, 2003).

En este enfoque de investigación, Creswell (2007) y González-Ugalde (2014), sostienen que se debe trabajar en grupo pequeños entre 5 y 25 participantes. Por esta razón, se entrevistó en profundidad a 10 estudiantes de cuarto de secundaria de una escuela pública de Lima-Perú. Estos estudiantes experimentaron durante el año académico 2018 el uso de una página de Facebook denominada "Entorno Virtual de Religión”. En ella, compartía información, participaban en debates, subían trabajos elaborados con recursos educativos de la web 2.0 y planteaban consultas y preguntas sobre las tareas y actividades desarrolladas en las clases. Cabe señalar que la implementación de esta página no fue obligatoria, pero al darse cuenta de que podían encontrar información importante para el desarrollo del curso y teniendo en cuenta que la mayoría tiene celular y accede a internet, el uso de la misma se generalizó rápidamente.

Para recoger la información, se usó una entrevista semiestructurada a partir de la técnica del auto-reporte. Así, se pudo indagar en profundidad la experiencia sobre un fenómeno particular (González-Ugalde, 2014 p.148). De esta forma se buscó descubrir formas cualitativamente diferentes en que las personas experimentan un fenómeno específico. En efecto, se elaboró una guía de entrevista a partir de las definiciones de las categorías y subcategorías implicadas y descritas en las investigaciones de Hofer y Pintrich (1997) para las creencias epistemológicas y Venkatesh, Thong y Xu (2003) para la UTAUT (cuadro 1). Las preguntas que se plantearon inicialmente fueron aplicadas con flexibilidad y dando espacios para repreguntas de acuerdo a las necesidades de comprensión de los estudiantes; buscando además que puedan reflexionar sobre su experiencia y tengan la libertad de responder con sinceridad (Creswell, 2007; González-Ugalde, 2014; Greasley y Ashwort, 2005; Linder y Marshall, 2003).

Cuadro 1. Definiciones de las subcategorías de estudio

\begin{tabular}{|c|c|c|c|}
\hline \multicolumn{2}{|c|}{ CREENCIAS EPISTEMOLÓGICAS } & \multicolumn{2}{|c|}{ ACEPTACIÓN Y USO DE LAS TECNOLOGÍAS } \\
\hline Subcategorías & Definición & Subcategorías & Definición \\
\hline $\begin{array}{l}\text { Certeza del } \\
\text { Conocimiento } \\
\text { (CEC) }\end{array}$ & $\begin{array}{c}\text { Grado en que un } \\
\text { conocimiento es entendido } \\
\text { como fijo o evoluciona en el } \\
\text { tiempo. }\end{array}$ & $\begin{array}{l}\text { Expectativa de } \\
\text { Rendimiento } \\
(\mathrm{PE})\end{array}$ & $\begin{array}{c}\text { Es el grado de las creencias } \\
\text { individuales sobre los beneficios } \\
\text { que puede obtener en el trabajo } \\
\text { con el uso de una tecnología. }\end{array}$ \\
\hline $\begin{array}{l}\text { Complejidad del } \\
\text { Conocimiento } \\
\text { (COC) }\end{array}$ & $\begin{array}{l}\text { Se refiere al hecho de que el } \\
\text { conocimiento es producto de } \\
\text { la acumulación de hechos y } \\
\text { conceptos altamente } \\
\text { interrelacionados. }\end{array}$ & $\begin{array}{l}\text { Expectativa de } \\
\text { Esfuerzo (EE) }\end{array}$ & $\begin{array}{l}\text { Es el grado de facilidad asociado } \\
\text { con el uso de una tecnología. }\end{array}$ \\
\hline $\begin{array}{l}\text { Fuente del } \\
\text { Conocimiento } \\
\text { (FUC) }\end{array}$ & $\begin{array}{c}\text { Se entiende como la } \\
\text { identificación del origen del } \\
\text { conocimiento sea en la } \\
\text { autoridad o en la } \\
\text { interrelación con los otros. }\end{array}$ & $\begin{array}{l}\text { Influencia Social } \\
\qquad(\mathrm{SI})\end{array}$ & $\begin{array}{c}\text { Es el grado en que un individuo } \\
\text { percibe que los demás creen que } \\
\text { debe usar una tecnología. }\end{array}$ \\
\hline $\begin{array}{l}\text { Justificación del } \\
\text { Conocimiento } \\
\text { (JC) }\end{array}$ & $\begin{array}{c}\text { Se refiere a la forma cómo } \\
\text { los individuos justifican sus } \\
\text { creencias basándose en } \\
\text { argumentos razonados y } \\
\text { evidencias. }\end{array}$ & $\begin{array}{c}\text { Facilidad de } \\
\text { Condiciones (FC) }\end{array}$ & $\begin{array}{c}\text { En el grado en que una persona } \\
\text { cree que una organización e } \\
\text { infraestructura técnica existen } \\
\text { para darle soporte al uso de una } \\
\text { tecnología. }\end{array}$ \\
\hline
\end{tabular}


Cabe señalar que en este tipo de investigaciones las categorías de estudio permiten la comprensión del fenómeno. De allí que no son exclusivas del punto de vista individual. Ahora bien, el trabajo de campo consistió en: (1) Seleccionar los grados de secundaria más adecuados para esta investigación; (2) Dialogar con el director de la I.E. para dar cuenta del estudio y las implicancias del mismo; (3) Seleccionar a los estudiantes de forma aleatoria, teniendo en cuenta su participación activa en el entorno virtual en cuestión; (4) Enviar cartas de consentimiento informado a los padres de familia a fin de solicitar el permiso respectivo y cumplir con la ética de la investigación; (5) Seleccionar a los estudiantes a partir de la aceptación de sus padres así como su compromiso y disponibilidad de tiempo.

Las entrevistas fueron realizadas entre los meses de octubre y noviembre del año 2018. Al finalizar se transcribieron asignándoles una etiqueta con el siguiente formato [E1, CECPE,4A, p.1]. En ella aparecen 4 elementos descriptores: (1) número identificativo de cada estudiante; (2) relación entre categorías a las que hace referencia el discurso, ej: certeza del conocimiento (CEC) y expectativa de rendimiento (PE); (3) el grado y sección a la que pertenecen; (4) la página de la transcripción donde se encuentra la cita. Con estos elementos ser procedió a seguir la siguiente ruta para el análisis (Trigwell, 2000; Willis, 2017). En primer lugar, se identificaron las palabras y expresiones más usadas por los estudiantes en relación a las categorías de estudio. Luego, se sistematizaron las expresiones seleccionadas de las entrevistas para organizar las categorías iniciales de significado. Acto seguido, se establecieron familias de términos para asignarles un significado conjunto y establecer las relaciones de categorías que los estudiantes habían manifestado. A continuación, se seleccionaron las citas más significativas de cada una de las categorías de trabajo con la intención de resaltar las relaciones entre las subcategorías del marco teórico. Finalmente, se identificaron las categorías finales y se escogieron las citas que coadyuvan al logro de los objetivos de la investigación.

\section{Resultados}

Los resultados se presentan teniendo en cuenta los objetivos de la investigación. En cuanto al primer objetivo, se puede evidenciar la existencia de relación entre las creencias epistemológicas y el nivel de aceptación y uso de la página de Facebook teniendo en cuenta los discursos de los estudiantes:

Porque ayuda bastante a las personas a desenvolverse, resolver bien los exámenes y sacar mejores notas. $Y$ además es gratis. [E4, CEC-PE, 4B, p. 3]

Sí, porque el docente publica información detallada sobre el tema que hemos tratado en clases. Entonces se me hace mucho más fácil buscar la información... Confío en lo que el docente me indica. [E5, COC-EE, 4A, p. 1]

Yo no usaba Facebook, recién lo comencé a usar en primero de secundaria porque mis amigos lo usaban. Es la moda entre todos. De ahí lo usé más porque el profesor comenzó a usarlo para actividades del curso. [E6, FUC-SI, 4C, p. 2]

Sí, a través de la página, por las encuestas. Sólo con dar like a la página siempre recibes las notificaciones y ya no te pierdes lo que faltó en clase... A mí lo que más me sirven son los videos, películas, cortometrajes. Eso me ayuda mucho a entender mejor el tema. [E1, JC-FC, 4B, p. 4]

Aunque consideran que: Facebook no siempre es seguro. A veces consigues información, pero no siempre es buena. [E2, JC-PE, 4B, p. 5] 
Estos fragmentos de discursos expresan la relación entre las categorías tal como se detalla en la figura 3.

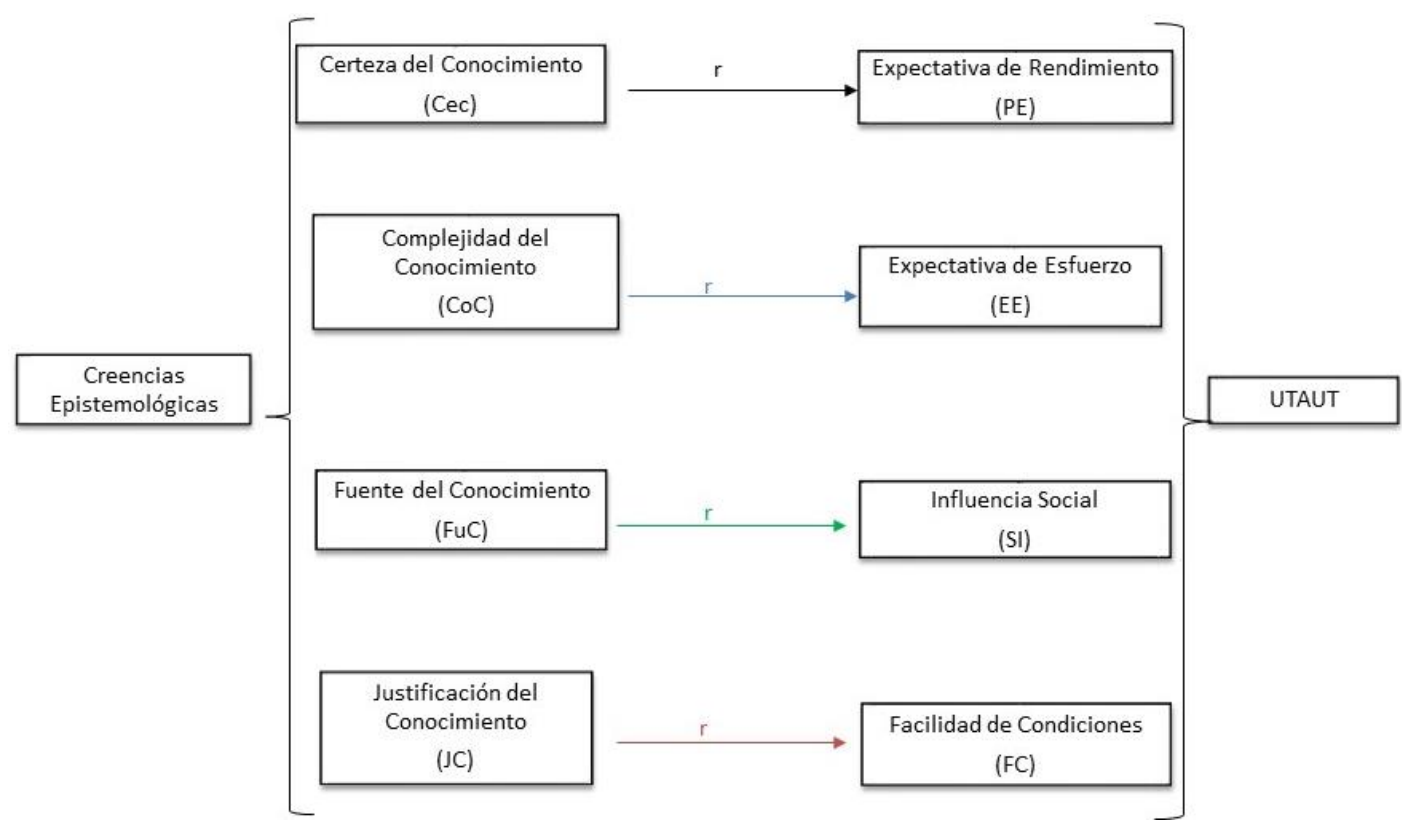

Figura 3. Categorías relacionadas en los discursos de los estudiantes Fuente: Elaboración propia.

Complementariamente a estos hallazgos, en las figuras 4a y 4b se puede apreciar el análisis de las entrevistas a la luz de las categorías estudiadas. Con esto, se confirma la aceptación y uso académico del entorno virtual de Facebook.

Respecto del segundo objetivo se pudo descubrir 6 categorías que expresan las razones de los estudiantes para aceptar y usar el entorno de Facebook. A continuación, se describen los hallazgos presentados en la figura 5 .

\subsection{Beneficios obtenidos con el uso de la página de Facebook}

Para los estudiantes, aceptar y usar Facebook está relacionado con creencias sobre: (1) existencia de grupos académicos; (2) complementar lo trabajado en clase; (3) obtener mejores notas en el curso; (4) ubicación de recursos de forma permanente; (5) aprender aspectos específicos del área. Así lo resaltan los siguientes discursos:

...hay grupos, canales que ayudan en cierta forma a aprender más de un tema... Hay un grupo de matemática donde estoy metido, los busqué porque estaba bajo en matemáticas. Busco grupos para aprender... por ejemplo un grupo de religión que me enseñó varias cosas que no sabía, me hizo entender más. [E8, FUC-PE, 4C, p. 29]

Facilita para seguir la secuencia de la clase. Es un complemento entre lo que veo y hago en clase. [E9, JC-FC, 4B, p. 36]

...puedo encontrar lo que necesito para resolver un examen o una prueba oral que el profesor me puede dejar. También puedo reforzar ideas o corregirme. Puede servirme como retroalimentación y así mejorar mis notas. [E4, JC-PE, 4B, p. 13]

... si falto un día, en la página de entorno virtual se encuentran los trabajos que deja. Puedo entrar y verificar que han dejado y darme una idea...Por ejemplo la película de Molokai que dejó e hizo preguntas en el aula. To la primera vez que se publicó no la vi y dio una segunda oportunidad y recién la pude ver para poder tener alguna participación en clase y sí ayuda mucho. [E1, JC-PE, 4B, p. 4] 
... la página ayuda a entender y aprender más. Así hago un mejor trabajo y mejoro mis notas... es más entendible, hay mayor información... por ejemplo, las lecturas de la Biblia fueron más entendibles con los videos... trabajos de manualidades.... [E7, COC-FC, 4C, p. 25]

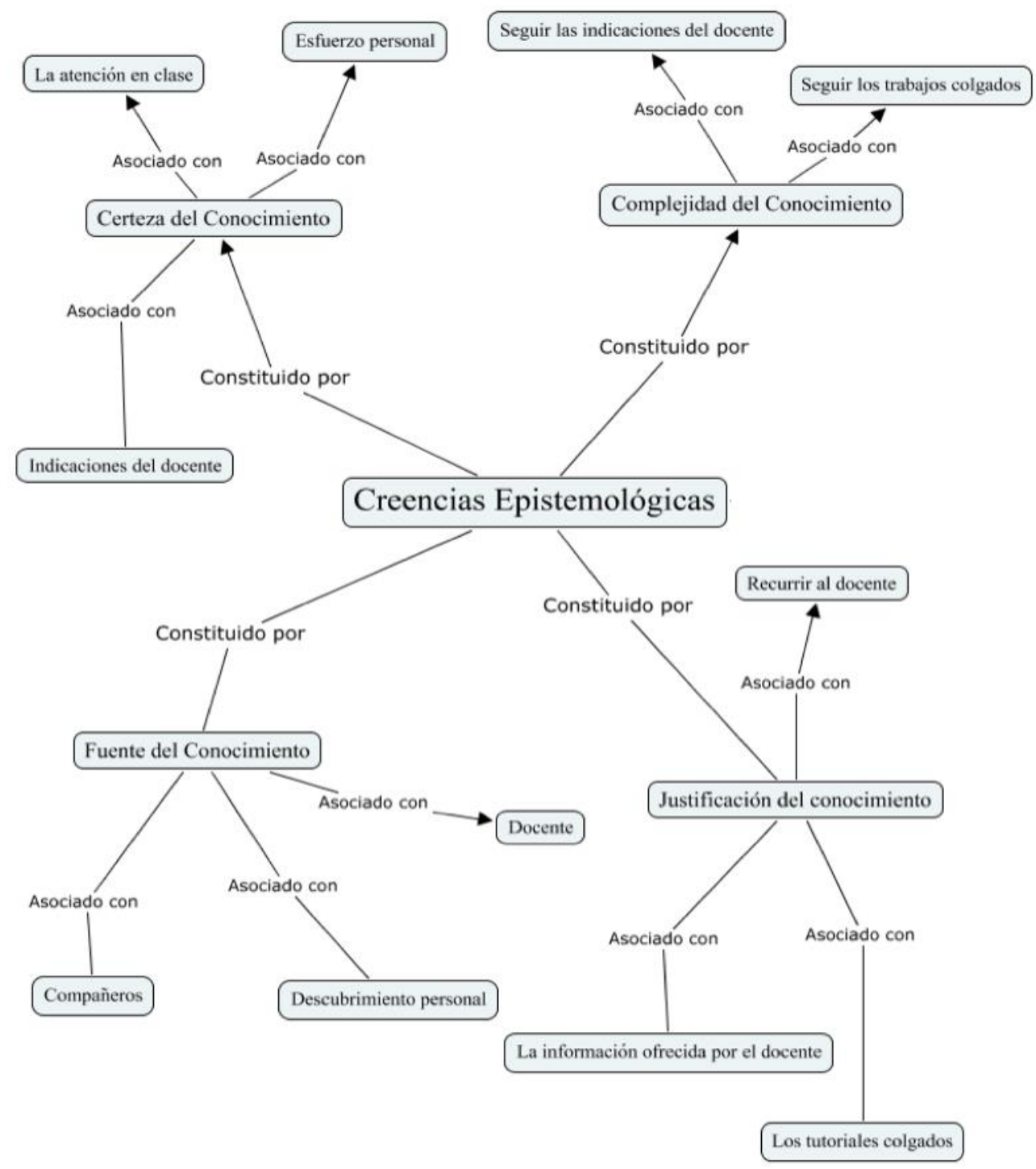

Figura 4a. Análisis de categorías y subcategorías de Creencias epistemológicas Fuente: Elaboración propia.

\subsection{Influencia social para aceptar usar la página de Facebook}

Los estudiantes indican que el uso de Facebook y la página diseñada para el curso en cuestión fue producto de dos grupos de personas: (1) adultos (docente y familiares) quienes les indicaron la utilidad y beneficios de la página y (2) compañeros de clase con los cuales se compartía intereses sociales y amicales.

Hace años cuando creé mi cuenta, mi hermana me enseñó cómo usarlo. Me dijo que era para comunicarme mejor. Ella me enseñó y confié en ella y luego vi que otros lo usaban y eso me animó a continuar en la red... Luego un profesor lo dijo. Es decir, siempre he visto la utilidad porque un adulto me lo ha recomendado. [E8, FUC-SI, $4 \mathrm{C}$, p. 30] 
Al principio vi que mis amigas lo tenían, por eso me creé uno. $\Upsilon$ de ahí lo usé para medios del colegio. Inicialmente fueron mis amigos. [E7, FUC-SI, 4C, p. 26]

Sin embargo, una participante añadió que si bien reconoce la influencia del docente en su decisión; contrasta las opiniones e incluso cuestiona al docente

Sí, mis compañeros me dijeron que usar Facebook es fácil, les creí porque los veía haciendo los trabajos, sus resultados y las notas. También el profesor y con ese uso veía los resultados y notas. Le doy más credibilidad a mi profesor. Pero también a mis compañeros. Contrasto lo dicho por el docente y mis compañeros. Pero cuestiono un poco a mi profesor. [E9, FUC-SI, 4B, p. 35]

Por otro lado, un grupo minoritario señala que el hecho de usar Facebook con fines de aprendizaje fue motivado por necesidades personales.

To lo usé desde un principio por comunicación, tenía a mi familia muy lejos y lo usaba para hablar con ellos. También, mantuve contacto con mis compañeros y además por educación, comencé a ver páginas que me informaban y fue por eso que empecé a usar Facebook. [E6, FUC-SI, 4A, p. 2]

No, yo lo he descubierto, y estoy convencido de eso. [E4, FUC-FC, 4B, p. 3]

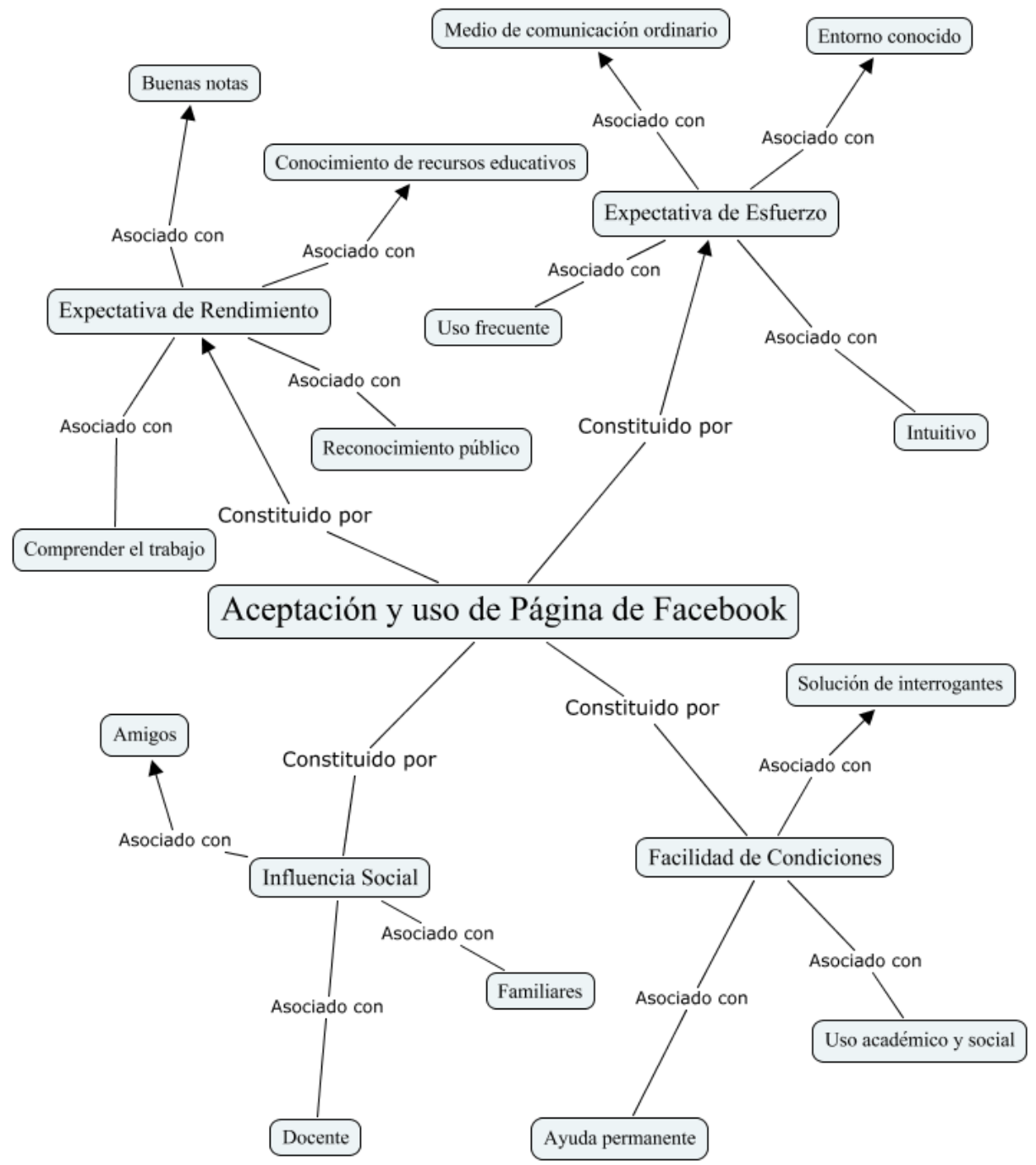

Figura 4b. Análisis de categorías y subcategorías de Aceptación y uso de página Facebook Fuente: Elaboración propia. 


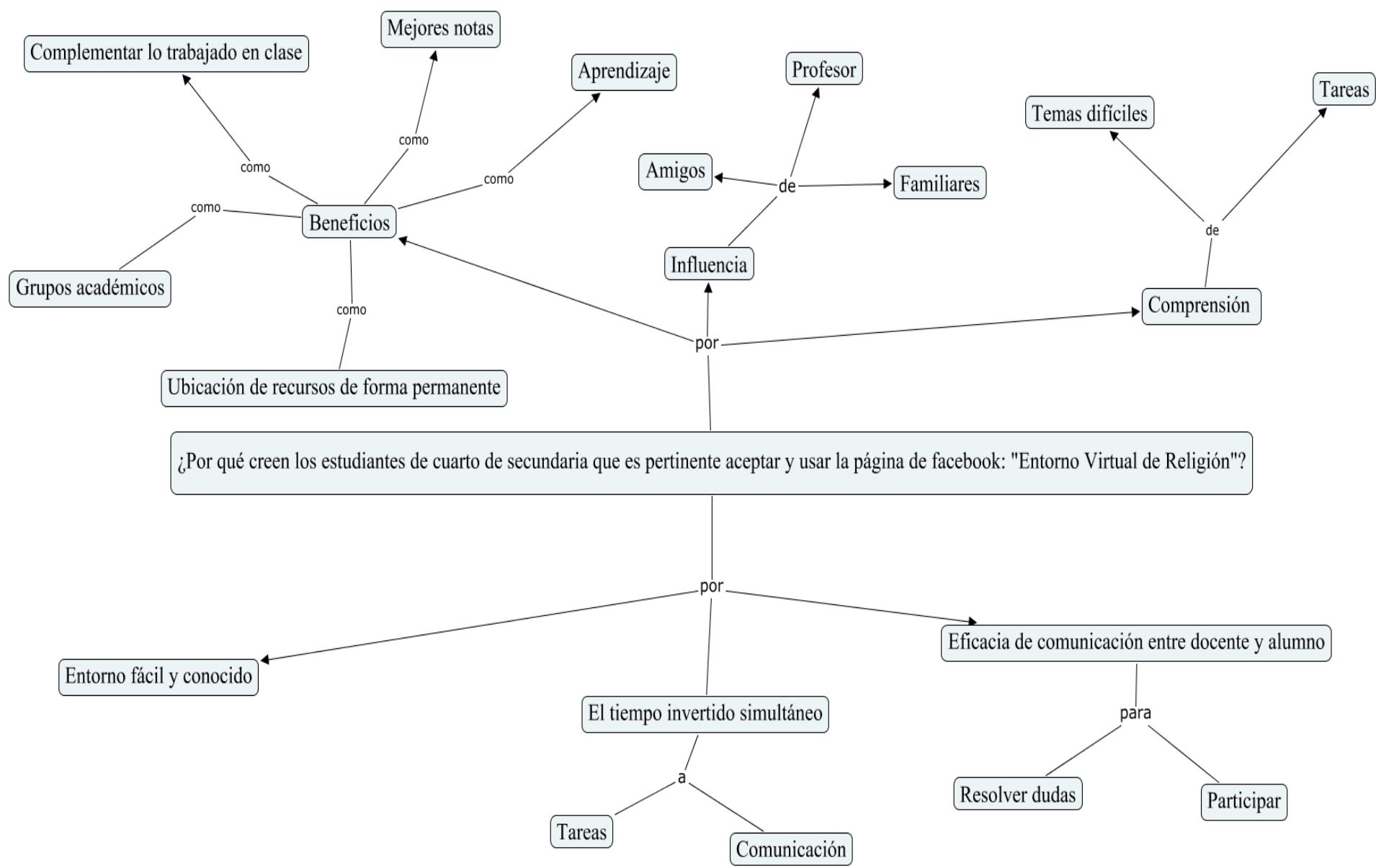

Figura 5. Sistema de creencias de los estudiantes a partir de las entrevistas realizadas

Fuente: Elaboración propia. 


\title{
3.3. Comprensión de actividades académicas
}

Básicamente los estudiantes consideran que el uso de Facebook se debe a que con los recursos y publicaciones del docente pueden entender los temas en desarrollo y los trabajos y actividades que se les plantea.

Es una manera de llegar a los alumnos, eso permite conocer lo que estamos haciendo en clases. Seguir la secuencia de lo que se hace en clase y así se puede estar en la línea de lo que el profesor espera. [E3, JC-FC, 4B, p. 11]

... al ver la página, hay mayor información y entiendo mejor. Se hace más fácil, hay información precisa... obtengo mayor información, entiendo mejor. Como publican videos es más llamativo y entendible. [E8, COC-EE, 4C, p. 30]

Cabe señalar que algunos estudiantes consideran que el beneficio también se relaciona con aspectos motivacionales y afectivos.

\begin{abstract}
Sí, porque puedes consultar dudas, publicar tus dudas y el profesor puede ver si el comentario es importante y tal vez ayude a aclarar otras dudas ya presentadas. $\Upsilon$ si es algo más específico puedes usar el inbox con el docente. Si lo recomendaría porque te puede ayudar mucho, se puede colocar también aspectos motivacionales, recomendaciones, no sólo tareas y actividades. [E2, JC-SI, 4B, p. 8]
\end{abstract}

\subsection{Entorno fácil y conocido}

La mayoría de estudiantes consideran que el entorno que ofrece la página de Facebook objeto de la investigación no exige una preparación o entrenamiento adicional. Por el contrario, es un entorno familiar y amigable. En efecto, antes del uso que se le da en clases, ellos lo usaban con fines sociales.
...todos tenemos Facebook en el colegio, es algo que en nuestro mundo es normal. Con la página todos pueden acceder. [E5, JC-EE, 4A, p. 19]
Al inicio fue algo complicado, porque no sabía cómo usarlo. Pero luego aparecieron tutoriales que te explicaban cómo usarlo. Lo principal era lo social, pero ahora puedes crear grupos, mandar mensajes, publicar fotos, puedes hacer casi de todo en Facebook. Es fácil porque ya lo conocía. Con el tiempo que uso Facebook se me ha hecho muy familiar. [E2, JC-EE, 4B, p. 7]

\subsection{Tiempo invertido en el uso de la página de Facebook}

En cuanto al tiempo la mayoría de estudiantes coincide en que el uso de la página de Facebook no requiere un tiempo adicional. Por el contrario, se adapta al tiempo de su uso regular con fines sociales al que están acostumbrados. En consecuencia, no constituye una recarga y no tienen dificultades en usar este entorno virtual con fines de aprendizaje.

Está en el tiempo normal de mi uso. Ingreso en un tiempo determinado y básicamente para revisar las páginas de Facebook así que no me genera tiempo adicional. Aprovecho también para comunicarme. [E7, JC-EE, 4A, p. 27]

... uno tranquilamente está en Facebook y te llega la notificación que el profesor ha publicado algo. Asi sabes que tienes que ver y hacer. [E10, JC-EE, 4A, p. 39]

\subsection{Eficacia de la comunicación entre docente y alumno}

En la última categoría los estudiantes consideran que el uso de la página de Facebook, ofrece la posibilidad de tener una comunicación eficiente con el docente. La efectividad se determina porque les permite aclarar dudas, consultar sobre las tareas y participar activamente, puesto que en las sesiones presenciales el tiempo no alcanza. 
...si tengo alguna duda puedo comunicarme con el docente. Quizás se demore en
contestar, pero nos resuelve la duda. No hay la excusa que no entendías. [E10, JC-EE,
$4 \mathrm{~A}, \mathrm{p} .40]$

Cabe señalar, que las herramientas de comunicación de la página también son usadas entre pares, aunque siempre apelan al prestigio del profesor al momento de despejar dudas.

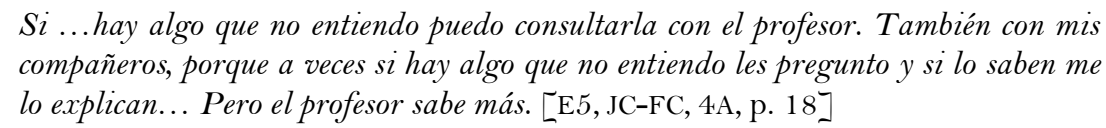

En este proceso, ellos también crean otros grupos para mantenerse en contacto y así ir aclarando las dudas, por lo que no se evidencia complicaciones en acepta y usar los servicios que Facebook ofrece.

\footnotetext{
...puedes comunicarte con tus profesores y compañeros. Si faltas puedes preguntarles a los compañeros o al docente. Hay grupos del salón a parte de la página del profesor. Es útil para comunicarme. [E2, CEC-FC, 4B, p. 6]
}

Finalmente, todos los estudiantes reconocen la utilidad y beneficios obtenidos con el uso de la página de Facebook en cuestión. Sin embargo, reflexionan sobre el tipo de uso que algunos le dan, los peligros existentes y el aprovechamiento de sus herramientas.

Es válido para aprender si le das un buen uso ya que también hay peligros con personas extrañas. [E5, JC-SI, 4A, p. 17]

...hay alumnos irresponsables que a pesar de tener las herramientas a la mano no las quieren usar... al final todo depende de cada uno. [E10, Coc-EE, 4A, p. 38]

\section{Discusión y conclusiones}

El desarrollo de la investigación ha permitido resaltar que la perspectiva del estudiante es fundamental para una enseñanza y aprendizaje efectivo (Stefl-Mabry, Radlick y Doane, 2010). De hecho, se constata que pocas investigaciones consideran las creencias de los estudiantes en la integración curricular de las tecnologías de la información y comunicación; deteniéndose más bien en la percepción de los maestros y gestores de la educación (Haixia, Chin-Hsi y Dongbo, 2017). Sin embargo, es sabido que el aprendizaje de los estudiantes mejora cuando los educadores prestan atención a conocimientos y creencias que los alumnos aportan a una tarea de aprendizaje y utilizan este conocimiento para desarrollar aprendizajes monitoreando sus percepciones a medida que el proceso de enseñanza continúa (Bransford, Brown y Cocking, 2000).

Ahora bien, como es sabido, la UTAUT constituye un aporte significativo en la creación de condiciones para la integración efectiva de tecnologías en la educación. Así, en los aproximadamente 15 años de vigencia ha sido aplicada de acuerdo a los requerimientos específicos de los investigadores (Lee, Korzan y Larsen, 2003; Venkatesh, Thong y Xu, 2016; Williams et al., 2011). En efecto, el interés ha sido impulsado por la constatación tanto teórica como empírica de que el éxito educativo de las herramientas tecnológicas depende del nivel de aceptación del usuario (Khechine, Lakhal y Ndjambou, 2016). Esta aceptación pasa necesariamente por lo que ellos creen que es útil, significativo y conveniente a su proceso de aprendizaje teniendo en cuenta la evolución de su pensamiento y madurez intelectual (Harteis, Gruber y Hertramph, 2010; Schommer, 1998). 
El primer objetivo planteaba comprender la relación entre las creencias epistemológicas de los estudiantes de cuarto de secundaria con la aceptación y uso de Facebook en el área de educación religiosa. Al respecto, está probado que efectivamente las creencias de las personas desempeñan un rol clave en el aprendizaje (Harteis, Gruber y Hertramph, 2010; Schommer, 1998) y la decisión de aceptación de las tecnologías con fines de aprendizaje (Haixia, Chin-Hsi y Dongbo, 2017; Halili y Sulaiman, 2018; Raman, Sani y Kaur, 2014; Teo, Doleck y Bazelais, 2018).

De hecho, los adolescentes están interesados en las tecnologías que proporcionan facilidad para el aprendizaje y crean experiencias divertidas y placenteras. Esto significa que sus creencias y expectativas son distintas a las de los adultos (Moghavvemi et al., 2017). En efecto, los participantes fueron mencionando que decidieron aceptar y usar Facebook por diversas razones, las cuales se asociaban a decisiones personales fruto del análisis y la reflexión o a la influencia de sus pares y del docente. Esto prueba lo planteado por Lee y Chan (2017), quienes afirman que las creencias epistemológicas están relacionadas con la metacognición y autorregulación. En otras palabras, la decisión de aceptar una tecnología parte de un proceso evolutivo y complejo que va configurando un significado particular para el usuario. Por tanto, las creencias de los estudiantes juegan un rol importante en relación a cómo afrontan el contenido didáctico y las tareas académicas de un área en particular (Pajares, 1992; Yilmaz-Tuzun y Sami, 2010), más aún si estos aspectos se encuentran potenciados con tecnología a través del entorno virtual de Facebook denominado en nuestro caso: "Entorno Virtual de Religión”.

En cuanto al segundo objetivo, se propuso describir las categorías finales que constituyen las creencias epistemológicas de los estudiantes en sus experiencias con el uso de Facebook en el área de educación religiosa. En este caso, se analizó los discursos de los alumnos desde las categorías planteadas por Hofer y Pintrich (1997) para las creencias epistemológicas y Venkatesh, Thong y Xu (2003) para la UTAUT. A partir del estudio, se encontraron 6 categorías que reflejan la relación entre las 8 sub categorías de las variables trabajadas por los autores mencionados. En la figura 6, se puede apreciar las relaciones existentes.

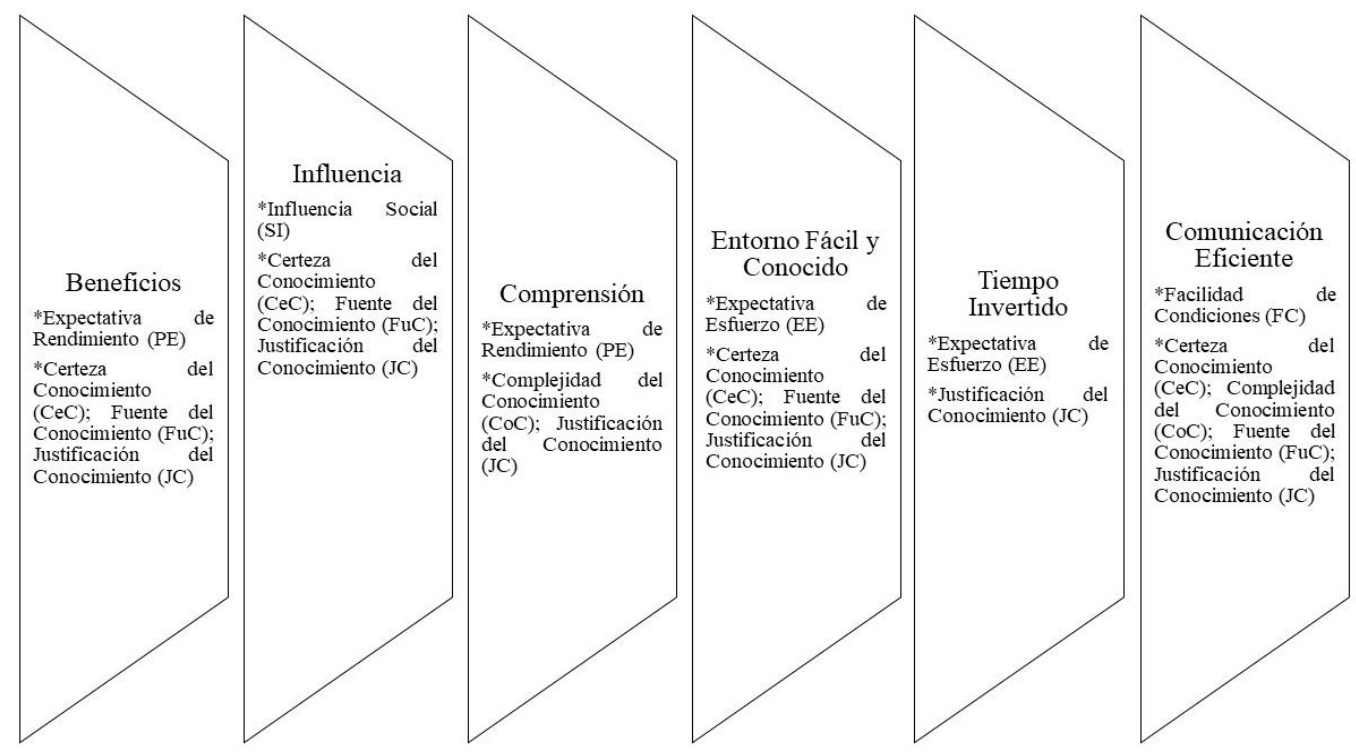

Figura 6. Categorías emergentes del discurso de los estudiantes Fuente: Elaboración propia. 
Estos hallazgos, reflejan un conjunto de creencias sobre cómo los estudiantes comprenden la utilidad de la página de Facebook "Entorno Virtual de Religión" desde la experiencia vivida a lo largo del año lectivo 2018. Lo cual es coherente con la metodología fenomenográfica, que "busca identificar y describir las formas cualitativamente diferentes sobre cómo las personas experimentan (comprenden, perciben) fenómenos de su entorno. Cabe señalar que estas experiencias no son consideradas como entidades físicas o mentales, sino como una relación entre objeto y sujeto". (González-Ugalde, 2014, p. 144).

Los resultados, coinciden con lo encontrado por Stefl-Mabry, Radlick y Doane (2010), quienes identificaron que los estudiantes utilizan de forma paralela los recursos tecnológicos para sus tareas y para la vida social. Así mismo, los alumnos entrevistados señalan que el uso de la página de Facebook "Entorno Virtual de Religión" no les representa un tiempo de trabajo adicional, sino que el tiempo invertido es el mismo para las tareas y la vida social. Por otro lado, Al-Azawei (2018) junto a Raman, Sani y Kaur (2014) encontraron que la expectativa de rendimiento constituye un factor de predictibilidad en el uso constante de Facebook, lo cual es congruente con la creencia de que el uso de la página "Entorno Virtual de Religión" ofrece beneficios académicos significativos. Esto, es complementado con el hecho de que Facebook ofrece un entorno familiar y fácil de usar, constituyendo así un factor de predictibilidad para su aceptación y uso (Venkatesh, 2000).

Además, con el estudio se identificó que la influencia social es un elemento determinante para la aceptación y uso de una tecnología. Así, Sánchez, Cortijo y Javed (2014) hallaron que la influencia social es el principal factor de adopción de Facebook; mientras que Aluri y Tucker (2015) sostienen que la influencia social determina las conductas de las personas. La red de influencias está formada por cuatro grupos: subordinados, compañeros, superiores y otros profesionales. En este caso los estudiantes participantes manifiestan que el uso de Facebook con fines de aprendizaje estuvo influenciado por sus compañeros y docente. Sin embargo, reconocen el nivel determinante de la autoridad del profesor por encima de sus pares. Esto también es coherente con el estudio de Carr y colaboradores (2013), quienes señalan que la credibilidad del instructor (docente, en este caso) afecta positivamente el conocimiento del área. De hecho, la influencia social determina una actitud positiva y accesible para cualquier innovación y desarrollo del conocimiento (Aluri y Tucker, 2015; Moghavvemi et al., 2017).

Para concluir se puede afirmar que: (1) Las creencias de los estudiantes constituyen factores significativos cuando se decide usar una tecnología en el proceso de enseñanzaaprendizaje; (2) Las creencias de los alumnos difieren de los adultos en función de su edad y experiencias adquiridas; (3) El marco teórico utilizado permitió analizar desde una perspectiva fenomenográfica las experiencias de los alumnos en el uso de Facebook en el área de educación religiosa; (4) Se lograron identificar seis categorías que describen las creencias epistemológicas de los alumnos participantes para aceptar y usar Facebook; y (5) Se puede inferir que la facilidad de uso, el tiempo invertido, la influencia social así como los beneficios académicos y de aprendizaje logrados en su experiencia escolar, constituyen las creencias de los alumnos para aceptar y usar la red social en cuestión.

Como limitaciones de la investigación, se puede considerar (1) la cantidad de estudiantes participantes. El diseño elegido implica trabajar con grupos pequeños por lo que sería conveniente desarrollar estudios similares con mayor cantidad de alumnos y en un enfoque mixto. Así, se podría recoger información cuantitativa que permita obtener 
tendencias y enriquecer el análisis e interpretación; (2) las dificultades de acceso a la red y equipos de cómputo disponibles en la institución educativa pública. Sin embargo, los estudiantes revisaban la información en sus celulares y trabajaban en sus hogares ya que la mayoría cuenta con dispositivos e internet.; y (3) las normas de la escuela que prohíben el uso de celulares y las redes sociales para evitar conductas ambiguas. Por ello se tuvo que informar a los padres de familia sobre esta metodología, así como solicitar el consentimiento informado y presentar un proyecto de trabajo a la dirección del plantel para obtener la autorización correspondiente.

Se sugiere realizar futuras investigaciones usar la teoría extendida UTAUT2 (Venkatesh, Thong y Xu, 2012) y en diferentes contextos escolares bajo un paradigma mixto. Así mismo, se debería estudiar las creencias epistemológicas de los estudiantes a lo largo del período escolar en las diferentes áreas curriculares para determinar su postura frente a los materiales y didáctica usada por los docentes. En este sentido, sería pertinente validar y estandarizar instrumentos basados en la teoría de Schommer $(1994,1998)$ para las creencias epistemológicas y de Venkatesh, Thong y Xu (2012) para la aceptación y uso de las tecnologías en educación. Finalmente, sería adecuado trabajar estas categorías de estudio en relación a otras tecnologías que los docentes usan para innovar y desarrollar competencias.

\section{Referencias}

Al-Azawei, A. (2018). Predicting the adoption of social media: An integrated model and empirical study on facebook usage. Interdisciplinary Journal of Information, Knowledge, and Management, 13, 233-257. https://doi.org/10.28945/4106

Aluri, A. y Tucker, E. (2015). Social influence and technology acceptance: The use of personal social media as a career enhancement tool among college students. Journal of Hospitality $\Xi^{2}$ Tourism Education, 27(2), 48-59. https://doi.org/10.1080/10963758.2015.1033103

Aypay, A. (2010). Teacher education student's epistemological beliefs and their conceptions about teaching and learning. Social and Behavioral Sciences, 2(2), 2599-2604. https://doi.org/10.1016/j.sbspro.2010.03.380

Belenky, M. F., Clinchy, B. M., Goldberger, N. R. y Tarule, J. M. (1986). Women's ways of knowing: The development of selfvoice and mind. Nueva York, NY: Basic Books

Bransford, J., Brown, A. y Cocking, R. (2000). How people learn. Washington, DC: National Academy Press.

Buehl, M. y Alexander, P. (2001). Beliefs about academic knowledge. Educational Psychology Review, 13(4) 385-418. https://doi.org/10.1023/A:1011917914756

Cano, F. y Cardelle-Elawar, M. (2004). An integrated analysis of secondary school students' conceptions and beliefs about learning. European Journal of Psychology of Education, 19(2), 167-187. https://doi.org/10.1007/BF03173230

Carr, C., Zube, P., Dickens, E., Hayter, C. y Barterian, J. A. (2013). Toward a model of sources of influence in online education: Cognitive learning and the effects of Web 2.0. Communication Education, 62(1), 61-85. https://doi.org/10.1080/03634523.2012.724535

Cvetkova, B., Atanasoska, T., Iliev, D., Andonovska-Trajkovska, D. y Seweryn-Kuzmanovska, M. (2015). Importance of investment in research's of students and teachers epistemological and pedagogical beliefs. Procedia-Social and Behavioral Sciences, 191, 1299-1303.

https://doi.org/10.1016/j.sbspro.2015.04.582 
Clark, C. y Peterson, P. (mayo, 1984). Teachers' thought processes. East Lansing, MI: Michigan State University.

Creswell, J. W. (2007). Qualitative inquiry and research design: Choosing among five approaches. Los Ángeles, CA: SAGE publications.

Davis, F. (1989). Perceived usefulness, perceived ease of use, and user acceptance of information technology. MIS Quarterly, 13(3), 319-340. https://doi.org/10.2307/249008

De Sena, R., Moriguchi, S. y Andrade, D. F. (2016). Intention of adoption of mobile payment: An analysis in the light of the unified theory of acceptance and use of technology (UTAUT). Revista de Administração e Inovação, 13(3), 22 1-230. https://doi.org/10.1016/j.rai.2016.06.003

Erickson, F. (2012). Qualitative research methods for science education. En Fraser, B. J. (Eds.), Second international handbook of science education (pp. 1451-1469). Dordrecht: Springer. https://doi.org/10.1007/978-1-4020-9041-7_93

Fishbein, M. y Ajzen, I. (1975). Belief, attitude, intention, and behavior: An introduction to theory and research. Reading, MA: Addison-Wesley

González-Ugalde, C. (2014). Investigación fenomenográfica. Revista Internacional de Investigación en Educación, 7(14), 141-158. https://doi.org/10.11144/Javeriana.M7-14.INFE

Haixia, L., Chin-Hsi, L. y Dongbo, Z (2017). Pedagogical beliefs and attitudes toward information and communication technology: A survey of teachers of English as a foreign language in China. Computer Assisted Language Learning, 30(8), 745-765. https://doi.org/10.1080/09588221.2017.1347572

Halili, S. H. y Sulaiman, H. (2018). Factors influencing the rural students' acceptance of using ICT for educational purposes. Kasetsart Journal of Social Sciences, 12, 1-6. https://doi.org/10.1016/j.kjss.2017.12.022

Harteis, C., Gruber, H. y Hertramph, H. (2010). How epistemic beliefs influence e-learning in daily work-life. Journal of Educational Technology E Society, 13(3), 201-211.

Hofer, B. y Pintrich, P. (1997). The development of epistemological theories: Beliefs about knowledge and knowing and their relation to learning. Review of Educational Research, 67(1), 88-140. https://doi.org/10.3102/00346543067001088

Khechine, H., Lakhal, S. y Ndjambou, P. (2016). A meta-analysis of the UTAUT model: Eleven years later. Canadian Journal of Administrative Sciences, 32(2), 138-152. https://doi.org/10.1002/cjas.1381

Kim, H. C. (2015). Acceptability engineering: The study of user acceptance of innovative technologies. Journal of Applied Research and Technology, 13(2), 230-237. https://doi.org/10.1016/j.jart.2015.06.001

Kizilgunes, B., Tekkaya, C. y Sungur, S. (2009). Modeling the relations among students' epistemological beliefs, motivation, learning approach, and achievement. The Journal of Educational Research, 102(4), 243-255. https://doi.org/10.3200/JOER.102.4.243-256

Lee, W. y Chan, V. C. (2017). Cross-sectional study on Chinese secondary school students' epistemic beliefs and fallacy identification. The Asia-Pacific Education Researcher, 26(1-2), 11 19. https://doi.org/10.1007/s40299-016-0322-1

Lee, Y., Kozar, K. y Larsen, K. (2003). The technology acceptance model: Past, present, and future. Communications of the Association for Information Systems, 12(1), 752-780.

https://doi.org/10.17705/1CAIS.01250 
Linder, C. y Marshall, D. (2003). Reflection and phenomenography: Towards theoretical and educational development possibilities. Learning and Instruction, 13(3), 271-284.

https://doi.org/10.1016/S0959-4752(02)00002-6

Lodewyk, K. (2007). Relations among epistemological beliefs, academic achievement, and task performance in secondary school students. Educational Psychology, 27(3), 307-327. https://doi.org/10.1080/01443410601104080

Ministerio de Educación. (2010). Orientaciones para el trabajo pedagógico. Lima: MINEDU.

Ministerio de Educación. (2015). Diseño curricular nacional de la educación básica regular. Lima: MINEDU.

Moghavvemi, S., Paramanathan, T., Rahin, N. y Sharabati, M. (2017). Student's perceptions towards using e-learning via Facebook. Behaviour \& Information Technology, 36(10), 10811100. https://doi.org/10.1080/0144929X.2017.1347201

Nassuora, A. B. (2012). Students acceptance of mobile learning for higher education in Saudi Arabia. American Academic E Scholarly Research Journal, 4(2), 24-30.

Pajares, F. (1992). Teachers' beliefs and educational research: Cleaning up a messy construct. Review of Educational Research, 62(3), 307-332. https://doi.org/10.3102/00346543062003307

Prieto, L. (2007). Autoeficacia del profesor universitario. Eficacia percibida y práctica docente. Madrid: Narcea.

Raman, A., Sani, R. M. y Kaur, P. (2014). Facebook as a collaborative and communication tool: A study of secondary school students in Malaysia. Procedia-Social and Behavioral Sciences, 155, 141-146. https://doi.org/10.1016/j.sbspro.2014.10.270

Sánchez, R., Cortijo, V. y Javed, U. (2014). Students' perceptions of Facebook for academic purposes. Computers E゚ Education, 70, 138-149.

https://doi.org/10.1016/j.compedu.2013.08.012

Samuelowicz, K. (1999). Academics' educational beliefs and teaching practices. Brisbane: Griffith University.

Schommer, M. (1994). Synthesizing epistemological belief research: Tentative understandings and provocative confusions. Educational Psychology Review, 6(4), 293-319. https://doi.org/10.1007/BFO2213418

Schommer, M. (1998). The role of adults' beliefs about knowledge and learning in school, work, and everyday life. En M. Smith y T. Pourchot (Eds.), Adult learning and development: Perspectives from educational psychology (pp. 127-143). Hillsdale, CA: Erlbaum.

Stefl-Mabry, J., Radlick, M. y Doane, W. (2010). ¿Can you hear me now? Student voice: High school \& middle school students' perceptions of teachers, ICT and learning. International Journal of Education and Development Using ICT, 6(4), 64-82.

Taherdoost, H. (2018). A review of technology acceptance and adoption models and theories. Procedia Manufacturing, 22, 960-967. https://doi.org/10.1016/j.promfg.2018.03.137

Teo, T., Doleck, T. y Bazelais, P. (2018). The role of attachment in Facebook usage: A study of Canadian college students. Interactive Learning Environments, 26(2), 256-272. https://doi.org/10.1080/10494820.2017.1315602

Trigwell, K. (2000). A phenomenographic interview on phenomenography. En J. Bowden y E. Walsh (Eds.), Phenomenography (pp. 62-82). Melbourne: RMIT University Press, 
Venkatesh, V. (2000). Determinants of perceived ease of use: Integrating control, intrinsic motivation, and emotion into the technology acceptance model. Information System Research, 11 (4), 342-365. https://doi.org/10.1287/isre.11.4.342.11872

Venkatesh, V., Thong, J. Y. L. y Xu, X. (2012). Consumer acceptance and use of information technology: Extending the unified theory of acceptance and use of technology. MIS Quarterly, 36(1), 157-178. https://doi.org/10.2307/41410412

Venkatesh, V., Thong, J. y Xu, X. (2016). Unified theory of acceptance and use of technology: A synthesis and the road ahead. Journal of the Association for Information Systems, 17(5), 328376. https://doi.org/10.17705/1jais.00428

Venkatesh, V., Morris, M. G., Davis, G. B. y Davis, F. D. (2003). User acceptance of information technology: Toward a unified view. MIS Quarterly, 27(3), 425-478.

https://doi.org/10.2307/30036540

Williams, M. D., Rana, N. P. y Dwivedi, Y. K. (2014). The unified theory of acceptance and use of technology (UTAUT): A literature review. Journal of Enterprise Information Management, 28(3), 443-488. https://doi.org/10.1108/JEIM-09-2014-0088

Williams, M., Rana, N., Dwivedi, Y. y Lal, B. (2011). Is UTAUT really used or just cited for the sake of it? a systematic review of citations of utaut's originating article. Recuperado de http://aisel.aisnet.org/ecis2011/231

Willis, A. S. (2017). The efficacy of phenomenography as a cross-cultural methodology for educational research. International Journal of Research \& Method in Education, 41(5), 483-499. https://doi.org/10.1080/1743727X.2017.1283398

Yilmaz-Tüzün, Ö. y Sami, M. (2010). Investigating the relationships among elementary school students' epistemological beliefs, metacognition, and constructivist science learning environment. Journal of Science Teacher Education, 21(2), 255-273. https://doi.org/10.1007/s 10972-009-9163-6

Zuiderwijk, A., Janssen, M. y Dwivedi, Y. K. (2015). Acceptance and use predictors of open data technologies: Drawing upon the unified theory of acceptance and use of technology. Government Information Quarterly, 32(4), 429-440. https://doi.org/10.1016/j.giq.2015.09.005

\section{Breve CV de los autores}

\section{Mario Armando Cartagena-Beteta}

Profesor de la Maestría en Integración e Innovación de las Tecnologías de la Información y la Comunicación en la Pontificia Universidad Católica del Perú. Magíster en Integración e Innovación Educativa de las Tecnologías de la Información y la Comunicación por la Pontificia Universidad Católica del Perú. Miembro del Grupo de Investigación Educación y Tecnología (EDUTEC-PUCP). Docente de Educación Religiosa en la I.E. 1264 "Juan A. Vivanco Amorín" (Lima, Perú). ORCID ID: https://orcid.org/0000-0002-1558-485X. Email: mcartagenab@pucp.pe

\section{Lucrecia Chumpitaz-Campos}

Profesora de la Escuela de Posgrado de la Universidad Femenina del Sagrado Corazón. Doctora de la Universidad Abierta de Catalunya (UOC). Miembro de la Sociedad de Investigación Educativa Peruana (SIEP). Miembro de World Educational Research 
REICE. Revista Iberoamericana sobre Calidad, Eficacia y Cambio en Educación

Association (WERA). ORCID ID: https://orcid.org/O000-0003-2454-8495. Email: lucreciachumpitazc@unife.edu.pe 